\title{
DIVULSIÓN DEL POLO INFERIOR: MÉTODO SEGURO Y EFICAZ PARA DISMINUIR LOS PARTOS ESPONTÁNEOS DESPUÉS DE LAS 41 SEMANAS
}

\author{
Enrique Valdés R. ${ }^{1}$, Paula Candia P. ${ }^{1}$, Rodrigo Terra V. ${ }^{1}$, Jaime Escobar D. ${ }^{1}$, Rafael \\ Caballero T. ${ }^{1}$, Guido Juarez D. ${ }^{1}$
}

${ }^{1}$ Departamento de Obstetricia y Ginecología, Hospital Clínico de la Universidad de Chile.

\begin{abstract}
RESUMEN
Objetivo: Conocer si la divulsión del polo inferior, realizada semanalmente desde las 38 semanas de gestación en pacientes de bajo riesgo, es un método seguro y eficaz para disminuir la incidencia de partos mayores de 41 semanas. Método: 110 embarazadas de bajo riesgo y de edad gestacional segura fueron randomizadas a divulsión de polo (casos) y no divulsión (controles). Desde las 38 semanas, tanto a las controles como las intervenidas, se les realizó tactos vaginales para evaluar pelvimetría, puntaje de Bishop y divulsión de polo en aquellas gestantes aleatorizadas y que cumplían las condiciones para poder realizar el procedimiento. El objetivo primario fue determinar la incidencia de parto espontáneo antes de las 41 semanas y el secundario fue evaluar la morbimortalidad materna y neonatal. Los datos fueron analizados por Chi cuadrado, test exacto de Fisher, test t de Student y test Wilcoxson rank-sum según correspondiera. Resultados: La población estudiada en ambos grupos fueron demográficamente similares. Las gestantes divulsionadas tuvieron significativamente $(p<0,005)$ menor cantidad de partos $(22,2 \%)$ después de las 41 semanas comparadas con el grupo de control (10,0\%). Las complicaciones materno-neonales fueron similares en ambos grupos. Conclusiones: La divulsión de polo es un método seguro y eficaz en disminuir la incidencia de partos después de las 41 semanas.
\end{abstract}

\section{PALABRAS CLAVES: Divulsión de polo, maduración cervical, embarazo en vías de prolongación}

\section{SUMMARY}

Objective: To determine whether weekly sweeping or stripping membranes beginning at 38 weeks could safely reduce the spontaneous labor rate after 41 weeks. Methods: 110 antenatal low-risk patients with firm gestational dates were randomized to either a treatment or control group. Control subjects received gentle cervical examination each week to asses Bishop score, whereas the treatment group also underwent weekly stripping of membranes beginning at 38 weeks. The primary outcome was the spontaneous labor rate before 41 weeks. Secondary outcomes included maternal and neonatal morbidity. The data were analyzed using Chi square, Fisher exact test, Student $t$ test and Wilcoxson rank-sum test. Results: The subjects were demographically similar between groups. Women who received treatment had fewer deliveries after 41 weeks than those in the control group $(22,2 \% \mathrm{v} / \mathrm{s} 10,0 \% ; p<0,05)$. Maternal- neonatal complications were similar in both groups. Conclusion: The stripping of membranes is a safe and effective method to reduce the incidence of labors after 41 week.

KEY WORDS: Membrane stripping, cervical maturation, prolonged pregnancy 


\section{INTRODUCCIÓN}

La divulsión del polo inferior, definido como el despegamiento digital de las membranas ovulares, es un método introducido en la práctica obstétrica desde hace unas veinte décadas por James Hamilton (1810) y desde entonces ha sido ampliamente usada, pero poco evaluada en su real beneficio. Este método ha sido utilizado con diversos fines en la práctica médica; quizás su principal indicación radica en la preeclampsia y en embarazos prolongados para lograr maduración cervical y obtener el desencadenamiento del parto en un breve lapso. A pesar de lo anteriormente citado los estudios realizados presentan conclusiones controversiales, ya que en algunos demuestran beneficio al lograr disminuir el embarazo prolongado y otros no (1).

El objetivo de este estudio es evaluar si la divulsión del polo inferior disminuye la incidencia del embarazo prolongado.

\section{MATERIAL Y MÉTODOS}

Estudio prospectivo, caso-control y randomizado (grupos permutados), realizado en 110 mujeres con embarazos fisiológicos, seleccionadas aleatoriamente según el orden dado por el grupo permutado. Se les efectuó desde las 38 semanas de amenorrea tacto vaginal con o sin divulsión del polo inferior según eran caso o control. Los cultivos corriente vagino-perineales efectuados a las 35 semanas eran negativos para gérmenes patógenos.

Rayburn y Chang (2) incluyen las 41 semanas o 287 días de gestación dentro del concepto de embarazo prolongado, y con el objetivo de disminuir la morbimortalidad perinatal, independiente de las condiciones cervicales, interrumpen el embarazo no importando el grado de madurez del cuello uterino. Nosotros hemos definido esa condición denominándola "embarazo en vías de prolongación" e indicamos la interrupción del embarazo previa prueba de tolerancia a las contracciones.

Para realizar la divulsión del polo inferior, además de tener cultivo vagino-perineales, es necesario tener un canal cervical entreabierto para poder realizar el despegamiento digital de la membrana corioamniótica con movimiento único circular, procedimiento que se repitió semanalmente hasta que el desencadenamiento del parto, teniendo como límite máximo las 41 semanas.

El objetivo primario de nuestro estudio es conocer si el procedimiento empleado disminuye la incidencia de parto después de la semana 41 , y el secundario establecer si se asocia a un incremento de la morbimortalidad materno-perinatal.

\section{RESULTADOS}

La edad gestacional promedio, la mediana del puntaje de Bishop, la edad gestacional promedio al parto, el peso del recién nacido y la paridad de las gestantes, no presentaron diferencias significativas entre los grupos estudio y control (Tabla I). La vía de parto, la rotura prematura de las membranas (RPM), la morbilidad febril materna y el puntaje de APGAR de los recién nacidos, no presentaron diferencias estadísticamente significativas; mientras que el grupo sometido a divulsión del polo inferior presenta una significativa disminución de la incidencia de partos después de las 41 semanas comparadas con el grupo control (10,0\% v/s 22,2\%; $p<0,05$ ) (Tabla II).

\section{DISCUSIÓN}

A pesar que la divulsión de polo inferior es una técnica utilizada desde hace 200 años, su uso se

Tabla I

CARACTERÍSTICAS DEMOGRÁFICAS Y OBSTÉTRICAS DE LA POBLACIÓN EN ESTUDIO

\begin{tabular}{llcr}
\hline Características & $\begin{array}{c}\text { Divulsión } \\
(n=47)\end{array}$ & $\begin{array}{c}\text { Controles } \\
(n=63)\end{array}$ & $p$ \\
\hline Edad materna* & $28,0 \pm 6,9$ & $27,7 \pm 5,3$ & $\mathrm{NS}^{\ddagger}$ \\
Puntaje de Bishop** & 5 & 3 & $\mathrm{NS}^{\dagger}$ \\
Puntaje de Bishop <7 (\%) & 94,2 & 88,2 & $\mathrm{NS}^{\#}$ \\
Edad gestacional al parto & $39,90 \pm 0,78$ & $39,48 \pm 1,55$ & $\mathrm{NS}^{\ddagger}$ \\
Peso nacimiento $(\mathrm{g})^{*}$ & $3538 \pm 339$ & $3598 \pm 362$ & $\mathrm{NS}^{\ddagger}$ \\
Nulípara $(\%)$ & 54,1 & 41,2 & $\mathrm{NS}^{\#}$ \\
\hline
\end{tabular}

"Promedio $\pm \mathrm{DE}$; ${ }^{* *}$ Mediana; NS: Diferencia no significativa; ${ }^{\star}$ Student $\mathrm{t}$ test; †Wilcoxon rank-sum test; \# $\mathrm{X}^{2}$ test. 
Tabla II

\section{ANÁLISIS COMPARATIVO DE LAS CARACTERÍSTICAS DE GESTANTES SOMETIDAS A DIVULSIÓN DE POLO INFERIOR Y CONTROLES}

\begin{tabular}{lccc}
\hline Características del parto & $\begin{array}{c}\text { Divulsión } \\
(n=47)\end{array}$ & $\begin{array}{c}\text { Controles } \\
(n=63)\end{array}$ & $p$ \\
\hline RPM (\%) & 14,8 & 17,4 & $\mathrm{NS}^{\#}$ \\
Morbilidad febril (\%) & 2,3 & 3,3 & $\mathrm{NS}^{\#}$ \\
Vías del parto (\%) & & & \\
Espontáneo & 53,9 & 53,0 & $\mathrm{NS} \#$ \\
Fórceps & 18,4 & 14,3 & $\mathrm{NS}^{\#}$ \\
Cesárea & 18,3 & 25,3 & $\mathrm{NS}^{\#}$ \\
$\begin{array}{l}\text { Parto espontáneo después } \\
\text { de las 41 semanas (\%) }\end{array}$ & 10,0 & 22,2 & $<0,05^{\ddagger}$ \\
Puntaje Apgar <7 (\%) & & & \\
1 min & 2,9 & 3,8 & $\mathrm{NS}^{\#}$ \\
5 min & 0 & 0 & - \\
\hline
\end{tabular}

NS: Diferencia no significativa; ${ }^{*}$ Fisher exact test; ${ }^{\ddagger}$ Student $t$ test.

ha transformado en una conducta rutinaria, no evaluándose su real efectividad en lograr una maduración cervical adecuada con el consiguiente parto espontáneo dentro de un breve lapso.

Fisiopatológicamente, la divulsión al despegar las membranas ovulares del polo inferior provocaría una deciduitis local, con liberación de una serie de mediadores de inflamación, entre otros, prostaglandinas y enzimas precursoras que inducirían la maduración cervical y el inicio del trabajo de parto (3).

Aunque en la literatura las conclusiones de la disminución de partos de postérmino y la menor cantidad en el número de inducciones en las pacientes a quienes se les realizó divulsión de polo no son definitivas $(4,5)$, existen evidencias que en pacientes con puntaje de Bishop desfavorables ( $\leq$ 5), se observó inicio del trabajo de parto espontáneo más a menudo que en los grupos sin intervención $(4,5,6)$, conclusión compartida por este estudio donde el embarazo postérmino y las inducciones disminuyeron en el grupo donde la divulsión se realizó.

Cabe destacar que en la población estudiada no hubo efectos iatrogénicos potenciales como son metrorragia y rotura uterina, y que las otras complicaciones como rotura prematura de membranas, corioamnionitis $y / 0$ infecciones maternas o del neonato no presentaron diferencias significativas entre los grupos.

Es importante destacar que la divulsión del polo inferior realizado semanalmente desde las 38 semanas, independiente del puntaje de Bishop, no aceleró la maduración cervical con el posterior comienzo del trabajo de parto dentro de la semana en que el procedimiento fue efectuado.

\section{CONCLUSIÓN}

La divulsión del polo inferior en embarazadas de bajo riesgo y libre de infección cérvico-vaginal es un método seguro y eficaz para lograr disminuir la incidencia de embarazos en vías de prolongación.

\section{BIBLIOGRAFÍA}

1. Adair CD. Nonpharmacologic approaches to cervical priming and labor induction. Clin Obstet Gynecol 2000; 43(3): 447-54.

2. Rayburn WF, Chang FE. Management of the uncomplicated postdate pregnancy. J Reprod Med 1981; 26: 93-97.

3. Mitchell MD, Flint AP, Bibby J, Brunt J, Arnold JM, Anderson $A B$, Turnbull AC. Rapid increases in plasma prostaglandin concentrations after vaginal examination and amniotomy. BMJ 1977; 2(6096): 1183-5.

4. McColgin SW, Patrissi GA, Morrison JC. Stripping the fetal membranes at term: Is the procedure safe and efficacious? J Reprod Med 1990; 35(8): 811-4.

5. Allott HA, Palmer CR. Sweeping the membranes: A valid procedure in stimulating the onset of labor? $\mathrm{Br}$ J Obstet Gynecol 1993; 100(10): 898-903.

6. Weissberg SM, Spellacy WN. Membrane stripping to induce labor. J Reprod Med 1977; 19: 125-7. 